\title{
Protective responses in the ischemic myocardium
}

\author{
R. Sanders Williams and Ivor J. Benjamin \\ Department of Internal Medicine, University of Texas Southwestern Medical Center, Dallas, Texas, USA \\ Address correspondence to: R. Sanders Williams, University of Texas Southwestern Medical Center, 6000 Harry Hines Boulevard, NB11.200, \\ Dallas, Texas 75390-8573, USA. Phone: (214) 648-1400; Fax: (214) 648-1450; E-mail: williams@ryburn.swmed.edu.
}

Acute coronary syndromes and heart failure arising as a consequence of ischemic injury to the myocardium account for a large proportion of all hospital admissions and of all causes of death in industrialized nations. Medical and surgical management of these conditions consumes enormous resources each year, but current therapeutic measures fall far short of reducing death and disability from ischemic heart disease to acceptable levels. Several recently successful advances in therapy of acute coronary syndromes are based on limiting the extent of myocardial damage that ensues following occlusion of a major coronary artery by rapid restoration of blood flow. However, many patients are not suitable candidates for thrombolytic drugs or revascularization procedures, and these approaches often are applied too late to prevent irreversible damage to the myocardium. A greater understanding of the mechanisms of ischemic injury, and of endogenous defense mechanisms, could foster additional improvements in clinical care.

\section{Mechanisms of cell injury during myocardial ischemia and reperfusion}

Cells of all tissues undergo irreversible injury and death when deprived of oxygen and other nutrients, and many of the responses to ischemia are shared among all cell types. However, quantitative and kinetic features of ischemic injury and of cytoprotective responses to injury are distinctive for each specialized cell type and tissue, and certain responses may be unique. In the case of the mammalian heart, an extensive literature has defined a stepwise series of events that follow coronary occlusion and that determine whether cells live or die.

Immediately upon cessation of blood flow, concentrations of high-energy phosphate compounds (ATP and creatine phosphate) in cardiomyocytes fall and $\mathrm{pH}$ rises as cells shift from oxidative metabolism to anaerobic glycolysis. Within seconds or minutes, these abnormalities become sufficiently severe to reduce the contractile force generated by actin-myosin crossbridge formation, and subsequently to impair the function of energy-dependent ion pumps in cellular membranes. Intracellular concentrations of free calcium increase, but cytosolic levels of calcium are buffered, at least initially, by uptake of calcium into mitochondria. As mitochondrial respiration ceases, FFAs and modified lipid or phospholipid intermediates accumulate.

These changes are reversible, without cell death, if blood flow is restored promptly. However, with increasing durations of ischemia, cardiomyocytes fail to maintain the integrity of cellular membranes and cell death becomes inevitable, even if blood flow is restored fully. The time course with which injury becomes irreversible is a function of many variables, including the species under study, the severity of ischemia (total versus partial), mechanical and metabolic demands on the heart, and the antecedent state of the myocardium. Clinical and experimental observations suggest that ischemic injury to the human myocardium may become irreversible within an hour, although there is clinical benefit to restoring blood flow even after longer periods of total coronary occlusion.

Restoration of coronary blood flow following a transient period of ischemia (reperfusion), though necessary for cell survival, introduces a separate series of stresses that can exacerbate cell injury. Reactive oxygen species (ROSs) generated during reperfusion damage proteins and membrane structures within cardiomyocytes and can activate signal transduction pathways that lead to apoptosis $(1,2)$. Adherence of leukocytes to postischemic endothelial cells both clogs capillaries (the "no reflow" phenomenon) and releases inflammatory mediators. Upon reperfusion, the influx of activated complement, catecholamines, and other signaling molecules contained in plasma or elaborated locally within the myocardial wall also may influence the course of events within cells of the myocardium. As with the direct consequences of ischemia, reperfusion injury is an important feature of acute coronary syndromes. Such injury occurs both spontaneously, as a result of fibrinolysis of coronary thromboses, and as a consequence of fibrinolytic drugs or acute angioplasty, treatments that are now commonly used to open occluded vessels.

At a molecular level, several classes of events provide final common pathways to cell death resulting from ischemia and reperfusion. Unfolding or pathological posttranslational modifications of critical proteins create a burden from which the cell cannot recover (3). Massive de novo resynthesis of proteins (see Bush et al., 
this Perspective series, ref. 4) carries a major energetic cost, and restoration of normal concentrations of key regulatory proteins may come too late to maintain the intracellular milieu in a manner consistent with survival. Events within ischemic cells also can target proteins for proteolytic destruction, and the accumulation of proteolytic fragments of certain proteins can have biological consequences (5). Damage to cell membranes, in addition to proteins, may preclude functional recovery. Calcium overload can impair recovery of mitochondrial function and can produce ischemic contractures with ensuing mechanical stresses, compounding the metabolic abnormalities (6). Finally, ischemia and reperfusion can trigger apoptosis, as a consequence of damage to mitochondria and leakage of cytochrome $c$, or through activation of receptormediated apoptotic signaling cascades (7).

\section{Cellular and molecular defenses against ischemic injury}

These mechanisms of ischemic cell injury in the heart are opposed by a diverse and remarkable set of cellular defenses that are induced during or after coronary occlusion and reperfusion. Most of these cytoprotective mechanisms are not unique to the heart, and certain stress responses are of ancient evolutionary origin and are conserved even in plants, yeast, and bacteria. Nevertheless, just as different mechanisms of ischemic cell injury assume lesser or greater importance in different cell types, cellular and molecular defense mechanisms also are deployed in a manner that, in quantitative and kinetic terms, is unique to myocardial cells.

Brief periods of transient ischemia reduce the extent of myocardial injury resulting from a subsequent and more prolonged ischemic episode. Of particular interest from both a biological and a clinical perspective is a phenomenon termed the ischemic preconditioning response. As noted originally in 1986 (8), the extent of myocardial infarction resulting from a sustained coronary occlusion is diminished if the heart has been subjected previously to brief periods of sublethal ischemia (i.e., preconditioning). A considerable literature describes efforts to understand the molecular basis for this phenomenon, and several signaling molecules have been found to play important roles. The literature on preconditioning is vast, and its interpretation is complicated by the diversity of experimental models and designs, and by different outcome measures (e.g., protection against infarction versus protection against stunning). Here we briefly summarize some general principles on which there seems to be a reasonable consensus.

The protective effects of a preconditioning stimulus are apparent within minutes and persist for days, but different mechanisms of cytoprotection take precedence in the early phase (first window of protection) and the late phase (second window of protection) of the preconditioning response. Within the early phase, cyto- protective mechanisms depend primarily on posttranslational modifications of pre-existing cellular proteins. Salutary effects in the later phases of the preconditioning response are mediated also by changes in gene expression and include events dependent on nitric oxide (NO) (9). Many signaling molecules have been implicated, but important pathways that mediate preconditioning responses are driven by adenosine-dependent activation of protein kinase $\mathrm{C} \delta$ (PKC $\delta$ ) or PKC $\varepsilon$ (10), resulting in activation of ATP-dependent potassium channels $\left(\mathrm{K}_{\mathrm{ATP}}\right)$ in mitochondria (11). Protein tyrosine kinases that activate $\mathrm{p} 42$ mitogen-activated protein kinase (p42MAPK) or p38MAPK also have been implicated (12) and may act upon the protein chaperone Hsp27 (13). In addition, ceramide, TNF- $\alpha$, or bradykinin activates $\mathrm{JNK}, \mathrm{NF}-\kappa \mathrm{B}$, and inducible nitric oxide synthase (iNOS). NO-dependent effects on $\mathrm{K}_{\mathrm{ATP}}$ channels $(1,14)$ have been shown in some models to function as signals that promote cytoprotective effects as components of the preconditioning response.

From the literature on preconditioning, as well as from other studies of biological mechanisms that limit ischemic injury in the heart, several general categories of cellular and molecular defenses can be described. Distinctions between these categories are not at all rigid, since there is substantial overlap and cross-talk among them, and these mechanistic distinctions may become blurred within the complex set of stresses presented to cells of the ischemic heart. Nevertheless, an attempt to categorize these cytoprotective responses remains useful in the effort to develop new therapeutic or preventive measures to enhance survival and recovery of cardiomyocytes following coronary occlusion.

Hypoxia-inducible factor 1 and its targets. An important defense mechanism is triggered directly by a deficiency of molecular oxygen, such that the ensuing adaptations render cells more capable of surviving and functioning within a hypoxic environment. The molecular mechanisms of this protective response are centered around the activation of hypoxia-inducible factor 1 (HIF-1), a heterodimeric transcription factor made up of HIF- $1 \alpha$ and aryl hydrocarbon nuclear translocator $(\mathrm{ARNT})(\mathrm{HIF}-1 \beta)$ proteins (see Semenza, this Perspective series, ref. 15). In normoxic cells, HIF- $1 \alpha$ is unstable, and few if any HIF-1 heterodimers are formed. During hypoxia, however, HIF- $1 \alpha$ is stabilized, leading to accumulation of the active HIF-1 $\alpha$ :ARNT heterodimer, which binds to specific recognition elements within promoter/enhancer regions of many target genes, the induction of which generates this cytoprotective process. The set of genes induced in this manner includes iNOS (16), which drives cytoprotective events medicated by NO. Other important classes of HIF-1 target genes in many cell types include those encoding glucose transporters and glycolytic enzymes, thereby enhancing the capacity for anaerobic production of high-energy phosphates, and angiogenic 
growth factors, which drive increased capillary density of the hypoxic tissue, serving to reduce the degree of hypoxia. In the heart, this defense mechanism is likely to function both in acute coronary syndromes (via NO generation) and in more stable adaptive responses to chronic or repeated bouts of ischemia. Several promising biotechnological strategies to enhance collateral blood flow to the ischemic heart are based on components of this hypoxic tolerance mechanism.

Protection from ROSs. Another defense mechanism is based on the increased synthesis of enzymes, driven by several different transcriptional control mechanisms, that allow the cell to reduce or detoxify ROSs. This cytoprotective mechanism is called into play in the preconditioning response, and transgenic animal models support the notion that an increased capacity to detoxify mediators of oxidative stress limits ischemic injury (17).

The mitochondrion in cytoprotective preconditioning. Other adaptive responses converge to protect the integrity of mitochondria, serving both to limit cytochrome $c$ release and activation of the terminal events of apoptosis, and to permit more rapid restoration of oxidative phosphorylation and ATP synthesis within cardiomyocytes once blood flow is restored to the myocardium. A number of signaling pathways triggered by IGF-1, cardiotrophin-1, FGF, PDGF, or TNF- $\alpha$ have been shown, at least under some conditions, to limit the extent of injury resulting from a period of coronary occlusion $(18,19)$. The downstream events that mediate these cytoprotective responses are complex but include the generation of antiapoptotic mediators that are targeted to mitochondria, as well as the modification of mitochondrial proteins such as $\mathrm{K}_{\text {ATP }}$ channels. Measures to reduce the degree of calcium overload by altering the activity of sarcolemmal or mitochondrial ion channels (11) or to stimulate anaerobic metabolism of citric acid cycle intermediates may also limit the extent of mitochondrial damage and dysfunction.

Induction of protein chaperones. Defense mechanisms based on the expression, activation, or redistribution of

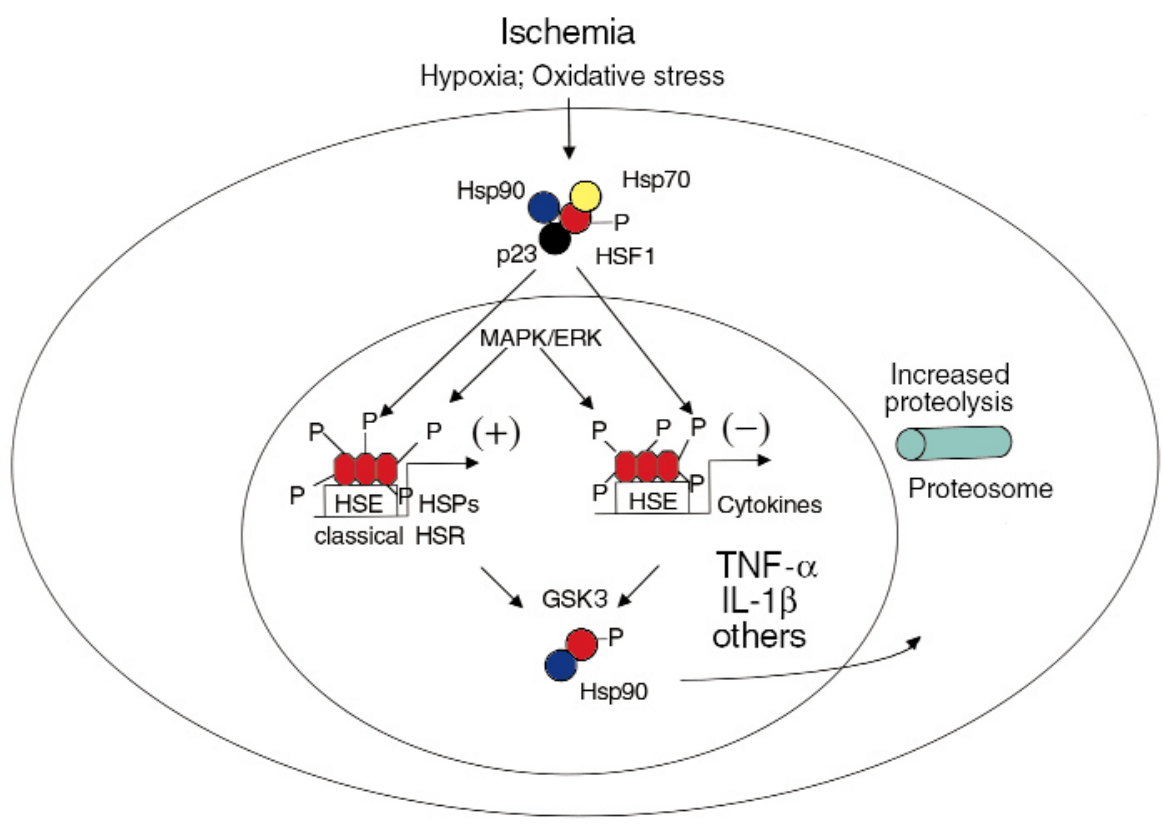

\section{Figure 1}

Heat shock transcription factor 1 (HSF1) has a central role in regulating transcription of genes important for cellular responses to ischemia and reperfusion. Under unstressed conditions, HSF1 is sequestered as an inactive monomer within a hetero-oligomeric complex containing Hsp90, Hsp70, FK506 binding protein 52, and p23 (33). Stimuli arising as a consequence of ischemia and reperfusion, which include ROSs, disrupt this complex and promote formation of HSF homotrimers, which bind to promoter/enhancer sequences of target genes. DNA binding of HSF1 exerts positive or negative effects on transcription of different sets of target genes, by means of recruitment of coactivator or corepressor proteins. HSF1 transactivates genes encoding heat shock proteins (HSPs), including those identified as components of the classical heat shock response (HSR). Full activation of HSF1 requires its phosphorylation by nuclear MAPK or extracellular signal-related kinase proteins, whereas phosphorylation by glycogen synthase kinase 3 (GSK3) has negative regulatory effects by promoting release of HSF1 from DNA and attenuation of the HSR. A recently described and complementary function for HSF1 is to suppress expression of proinflammatory cytokines such as TNF- $\alpha$ and IL-1 $\beta$ in vitro and in vivo (36). It has not been established whether the function of HSF1 as a negative regulator is restricted to specific cell types (e.g., blood-borne monocytes and macrophages). HSF1 is essential for activation of the protein chaperone defense stimulated by thermal stress (29) or by other conditions (e.g., inhibition of proteosome function) that increase the concentration of unfolded proteins within the cell (37). HSE, heat shock element. P, phosphorylated amino acid residues. 
molecular chaperone proteins counteract one of the most fundamental mechanisms of ischemic injury, the unfolding, misfolding, or pathological modification (e.g., peroxidation) of critical proteins in various intracellular compartments. Conformational alterations of individual proteins in ischemic cells become manifest rapidly at higher levels of organization as rearrangements of cytoskeletal structures and organelles, and as physiological dysfunction, ultimately threatening cell survival. All cells have evolved distinct molecular pathways to limit or to reverse the effects of protein unfolding or misfolding, and these defense mechanisms are strategically positioned within major subcellular compartments such as the cytoplasm, mitochondria, peroxisomes, nucleus, and endoplasmic reticulum. In cardiac myocytes, specialized stress proteins also are associated with sarcomeric structures (3). This category of defense mechanism within the ischemic heart shares many features with the heat shock response, one of the most ancient and conserved countermeasures by which cells of all organisms respond to environmental stress.

The heat shock response is conserved from bacteria to humans (20), and it represents a fundamental endogenous mechanism to antagonize protein unfolding or misfolding. A distinct but related pathway termed the "unfolded protein response" has been recently described for handling protein misfolding specifically within the endoplasmic reticulum, a major site for processing of membrane-bound and secreted proteins (21). A central feature of the heat shock response that is shared with the unfolded protein response, as well as with the aforementioned cellular response to hypoxia, is activation of a specific transcription factor, the function of which is tightly regulated in response to stress conditions encountered during ischemia/reperfusion.

Individual genes that are the downstream targets of the heat shock response pathway include several members of the heat shock protein (HSP) multigene family. Many of the proteins encoded by these genes function as molecular chaperones to promote the folding and assembly of nascent polypeptides, and to facilitate the repair or degradation of unfolded proteins (22). The major inducible stress protein $\mathrm{Hsp} 70$ directly protects against myocardial ischemic damage, improves metabolic recovery, enhances functional recovery, and reduces infarct size in hearts of transgenic mice (24). Likewise, reversible damage after simulated or myocardial ischemia can be mitigated by overexpression of the small HSPs $\alpha$ B-crystallin $(3,25)$ and Hsp27 (14).

In addition to their functions as molecular chaperones, HSPs may act as signaling molecules. A recent report describes a cytokine-like function for Hsp70 in human monocytes (26). Increased expression of TNF$\alpha$, IL-1, and IL- 6 was stimulated by high-affinity binding of Hsp70 to plasma membrane receptors that include CD14. Upregulation of target genes by this stimulus was attributable both to an increase in intracellular calcium and to activation of NF- $\mathrm{KB}$. The function of Hsp70 as a signaling molecule, in addition to its role as protein chaperone, may prove to be important in the context of ischemic injury to the myocardium.

\section{Transcriptional control of the protein chaperone defense}

Synthesis of HSPs is rapidly induced in response to several forms of cell stress, including hyperthermia, hypoxia, ischemia, and mechanical stretch applied to cells or intact organs (27). This response requires heat shock transcription factor 1 (HSF1), the primary mediator of the heat shock response (Figure 1). Under stressful conditions, homotrimers of HSF1 bind to triplet repeats of a nucleotide recognition motif (nGAAn) within promoter/enhancer regions of downstream target genes that encode the classical HSPs, and other proteins as well. Three other heat shock transcription factor isoforms (HSF2-4), products of distinct genes $(28,29)$, are present in mammalian cells but are not sufficient to preserve the heat shock response in the absence of HSF1 (30). Disruption of the Hsf1 gene in mice eliminates the heat shock response, abolishes acquired thermotolerance in vitro, and increases the susceptibility of HSF1-deficient cells to heatinduced apoptosis (30). It is likely that stress-inducible expression of HSPs mediated by HSF1 plays a key role in protective responses to myocardial ischemia, including the late preconditioning response, and this premise is under active investigation using $H s f 1$ knockout mice. An impairment in the heat shock response during ischemia, in association with decreased DNA binding by HSF1, has been observed in hearts of older animals as compared with young animals. This age-related change may contribute to an increased vulnerability to ischemic injury in elderly individuals.

HSF1 activation during ischemia. Binding of HSF1 to its recognition element within DNA is increased in cells subjected to conditions that simulate tissue ischemia (30). A decrease in the concentration of high-energy phosphate compounds appears to be sufficient to evoke this response (31), but intracellular acidosis may serve as an additional stimulus. Using a novel in vitro system, Wu and coworkers demonstrated direct initiation of HSF1 trimerization leading to full transcriptional potency by increased temperature and/or oxidative stress (32). Other chemical inducers that activate the heat shock response in intact cells (e.g., alcohol, salicylate, and arsenite) were incapable of activating HSF1 in vitro. A common consequence of stimuli that activate HSF1 is an increase in the concentrations of unfolded proteins within the cell, which may provide a proximate stimulus for induction of HSP gene expression.

HSF1 suppression under unstressed conditions. Several regulatory mechanisms have been shown to repress DNA binding and transcriptional activation by HSF1 in 
unstressed cells. Structural studies indicate that eukaryotic HSF1 is organized into functional domains, beginning with a conserved DNA-binding region at the $\mathrm{NH}_{2}-$ terminal, an adjacent oligomerization domain containing leucine zipper motifs, a nuclear localization signal, and a $\mathrm{COOH}$-terminal transactivation domain (27). Although HSF1 is present in the nucleus of unstressed cells, this regulator is held in an inactive conformation by strong intramolecular interactions involving a COOH-terminal hydrophobic repeat domain. In addition, in unstressed cells HSF1 forms a complex in association with Hsp90, Hsp/c70, FK506 binding protein 52 , and $\mathrm{p} 23$ proteins (33). Hetero-oligomeric complexes in which HSF1 is bound to Hsp90 may provide a conformational switch required for rapid activation of HSF1 in a manner that mimics the kinetics of steroid receptor pathways mediated by cochaperones. Finally, phosphorylation of HSF1 provides additional regulatory control over its functional activity. Both MAPK and extracellular signal-related kinase and glycogen synthase kinase (GSK) activities are induced by ischemia and inflammatory cytokines and alter the phosphorylation state of HSF1. In particular, increased expression of GSK attenuates HSF1 activity and reduces the duration of the heat shock response (34).

Transcriptional repression of inflammatory responses. In human monocytes, activation of HSF1 correlates with suppression of proIL- $1 \beta$ transcription after LPS stimulation. This reduction in cytokine production is associated with increased DNA-binding activity of HSF1, as assessed by electrophoretic mobility shift assays using a sequence element from the IL1 $\beta$ promoter. A dose-dependent suppression of TNF $\alpha$ promoter activity, accompanied by increased DNA binding of HSF1, also is produced in response to LPS stimulation of macrophages (35). TNF- $\alpha$ production in response to endotoxin is significantly greater in intact animals devoid of HSF1 ( $\mathrm{Hsf1}^{-/-}$mice) compared with heterozygous controls, and the exaggerated production of TNF- $\alpha$ in $H s f 1^{-/}$mice after endotoxin challenge is associated with increased mortality (36). These recent studies reveal a previously unsuspected role for HSF1 as a negative transcriptional regulator in vivo, in addition to its well established function in transactivation of genes encoding cytoprotective HSPs.

Other physiological roles for mammalian HSF1. Mammalian HSFs have been proposed to serve unique physiological functions during development and reproduction of complex organisms (27). $\mathrm{Hsf1}^{-/-}$mice survive to adulthood but exhibit multiple phenotypes that include female infertility, placental defects, and postnatal growth retardation, even under presumptively unstressed conditions. Expression of HSF2, which shares $40 \%$ sequence identity with HSF1, is unimpaired by $H s f 1$ inactivation, but this regulator does not rescue the heat shock response (29). However, extraembryonic abnormalities caused by HSF1 inactivation are dis- sociated from appreciable changes in the expression of major HSPs (36), suggesting that HSF1 functions to control transcription of other genes not currently identified as HSPs. This principle may prove pertinent to the myocardium as well.

\section{Implications for medical therapeutics}

Our understanding of cellular defense mechanisms that limit myocardial injury following ischemia and reperfusion has progressed markedly over the past several years. In particular, we can now point with confidence to a number of specific signaling molecules and terminal effectors of pathways that exert important cytoprotective functions. Additional mechanisms of cellular defense remain to be identified, and many features of the known pathways have not been fully elucidated. Nevertheless, current knowledge provides a reasonable base of information on which novel pharmacologic or genetic countermeasures to ischemic injury can be rationally based. Recent editorials in prominent cardiovascular journals call for an aggressive program of clinical research to this achieve this goal. Translating mechanistic information into clinically relevant therapeutic or preventive strategies is no small task and is complicated by the distinct potential of certain cytoprotective mechanisms to have deleterious consequences if stimulated in excess. Nevertheless, it seems likely that meaningful new therapies can emerge from this promising field of investigation.

\section{Data supplement}

Due to space constraints, a number of important references could not be included below. Interested readers can find a supplementary reading list at: http://www.jci.org/cgi/content/full/106/7/813/DC1.

\footnotetext{
1. Hernandez, O.M., Discher, D.J., Bishopric, N.H., and Webster, K.A. 2000. Rapid activation of neutral sphingomyelinase by hypoxia-reoxygenation of cardiac myocytes. Circ. Res. 86:198-204.

2. Semenza, G.L. 2000. Cellular and molecular dissection of reperfusion injury: ROS within and without. Circ. Res. 86:117-118.

3. Xiao, X., and Benjamin, I.J. 1999. Stress-response proteins in cardiovascular disease. Am. J. Hum. Genet. 64:685-690.

4. Bush, K.T., Keller, S.H., and Nigam, S.K. 2000. Genesis and reversal of the ischemic phenotype in epithelial cells. J. Clin. Invest. 106:621-626.

5. Murphy, A.M., et al. 2000. Transgenic mouse model of stunned myocardium. Science. 287:488-491

6. Wang, Y., and Ashraf, M. 1999. Role of protein kinase C in mitochondrial KATP channel-mediated protection against $\mathrm{Ca} 2+$ overload injury in rat myocardium. Circ. Res. 84:1156-1165.

7. Bialik, S., et al. 1999. The mitochondrial apoptotic pathway is activated by serum and glucose deprivation in cardiac myocytes. Circ. Res. 85:403-414.

8. Murry, C.E., Jennings, R.B., and Reimer, K.A. 1986. Preconditioning with ischemia: a delay of lethal cell injury in ischemic myocardium. Circulation. 74:1124-1136.

9. Guo, Y., et al. 1999. The late phase of ischemic preconditioning is abrogated by targeted disruption of the inducible NO synthase gene. Proc. Natl. Acad. Sci. USA. 96:11507-11512.

10. Zhao, J., et al. 1998. The expression of constitutively active isotypes of protein kinase $\mathrm{C}$ to investigate preconditioning. J. Biol. Chem. 273:23072-23079.

11. Sato, T. 1999. Signaling in late preconditioning: involvement of mitochondrial K(ATP) channels. Circ. Res. 85:1113-1114.

12. Dawn, B., et al. 1999. Bifunctional role of protein tyrosine kinases in late
} 
preconditioning against myocardial stunning in conscious rabbits. Circ. Res. 85:1154-1163.

13. Dana, A., Skarli, M., Papakrivopoulou, J., and Yellon, D.M. 2000. Adenosine $\mathrm{A}(1)$ receptor induced delayed preconditioning in rabbits: induction of 338 mitogen-activated protein kinase activation and Hsp27 phosphorylation via a tyrosine kinase- and protein kinase C-dependent mechanism. Circ. Res. 86:989-997.

14. Sasaki, N., Sato, T., Ohler, A., O’Rourke, B., and Marban, E. 2000. Activation of mitochondrial ATP-dependent potassium channels by nitric oxide. Circulation. 101:439-445.

15. Semenza, G.L. 2000. Surviving ischemia: adaptive responses mediated by hypoxia-inducible factor 1. J. Clin. Invest. 106:809-812.

16. Jung, F., Palmer, L.A., Zhou, N., and Johns, R.A. 2000. Hypoxic regulation of inducible nitric oxide synthase via hypoxia inducible factor- 1 in cardiac myocytes. Circ. Res. 86:319-325.

17. Wang, P., et al. 1998. Overexpression of human copper, zinc-superoxide dismutase (SOD1) prevents postischemic injury. Proc. Natl. Acad. Sci. USA. 95:4556-4560.

18. Matsui, T., et al. 1999. Adenoviral gene transfer of activated phosphatidylinositol 3'-kinase and Akt inhibits apoptosis of hypoxic cardiomyocytes in vitro. Circulation. 100:2373-2379.

19. Kurrelmeyer, K.M., et al. 2000. Endogenous tumor necrosis factor protects the adult cardiac myocyte against ischemic-induced apoptosis in a murine model of acute myocardial infarction. Proc. Natl. Acad. Sci. USA. 97:5456-5461.

20. Lindquist, S., and Craig, E.A. 1988. The heat-shock proteins. Annu. Rev. Genet. 22:631-677.

21. Mori, K. 2000. Tripartite management of unfolded proteins in the endoplasmic reticulum. Cell. 101:451-454.

22. Gething, M.J., and Sambrook, J. 1992. Protein folding in the cell. Nature. 355:33-45.

23. Radford, N.B., et al. 1996. Cardioprotective effects of 70-kDa heat shock protein transgenic mice. Proc. Natl. Acad. Sci. USA. 93:2339-2342.

24. Martin, J.L., Mestril, R., Hilal-Dandan, R., Brunton, L.L., and Dillmann, W.H. 1997. Small heat shock proteins and protection against ischemic injury in cardiac myocytes. Circulation. 96:4343-4348.

25. Asea, A., et al. 2000. HSP70 stimulates cytokine production through a CD14-dependent pathway, demonstrating its dual role as a chaperone and cytokine. Nat. Med. 6:435-442.
26. Benjamin, I.J., Kroger, B., and Williams, R.S. 1990. Activation of the heat shock transcription factor by hypoxia in mammalian cells. Proc. Natl. Acad. Sci. USA. 87:6263-6267.

27. Wu, C. 1995 . Heat shock transcription factors: structure and regulation. Annu. Rev. Cell Dev. Biol. 11:441-469.

28. Morimoto, R.I. 1998. Regulation of the heat shock transcriptional response: cross talk between a family of heat shock factors, molecular chaperones, and negative regulators. Genes Dev. 12:3788-3796.

29. McMillan, D.R., Xiao, X., Shao, L., Graves, K., and Benjamin, I.J. 1998. Targeted disruption of heat shock transcription factor 1 abolishes thermotolerance and protection against heat-inducible apoptosis. J. Biol. Chem. 273:7523-7528.

30. Benjamin, I.J., and McMillan, D.R. 1998. Stress (heat shock) proteins: molecular chaperones in cardiovascular biology and disease. Circ. Res. 83:117-132.

31. Benjamin, I.J., Horie, S., Greenberg, M.L., Alpern, R.J., and Williams, R.S. 1992. Induction of stress proteins in cultured myogenic cells. Molecular signals for the activation of heat shock transcription factor during ischemia. J. Clin. Invest. 89:1685-1689.

32. Zhong, M., Orosz, A., and Wu, C. 1998. Direct sensing of heat and oxidation by Drosophila heat shock transcription factor. Mol. Cell. 2:101-108.

33. Zou, J., Guo, Y., Guettouche, T., Smith, D.F., and Voellmy, R. 1998. Repression of heat shock transcription factor HSF1 activation by HSP90 (HSP90 complex) that forms a stress-sensitive complex with HSF1. Cell. 94:471-480.

34. Xavier, I.J., et al. 2000. Glycogen synthase kinase 3b negatively regulates both DNA-binding and transcriptional activities of heat shock factor 1 . J. Biol. Chem. 275:29147-29152.

35. Singh, I.S., Viscardi, R.M., Kalvakolanu, I., Calderwood, S., and Hasday, J.D. 2000. Inhibition of tumor necrosis factor-alpha transcription in macrophages exposed to febrile range temperature. A possible role for heat shock factor-1 as a negative transcriptional regulator. J. Biol. Chem. 275:9841-9848

36. Xiao, X., et al. 1999. HSF1 is required for extra-embryonic development, postnatal growth and protection during inflammatory responses in mice. EMBO J. 18:5943-5952.

37. Pirkkala, L., Alastalo, T.P., Zuo, X., Benjamin, I.J., and Sistonen, L. 2000. Disruption of heat shock factor 1 reveals an essential role in the ubiquitin proteolytic pathway. Mol. Cell. Biol. 20:2670-2675. 